\title{
Bulbous Tip Correction Focusing on Skin Soft Tissue Envelope in Asian Rhinoplasty
}

\author{
Da Arm Kim ${ }^{1,5}$, Jae Yong Jeong ${ }^{2,3,5}$, \\ Sang $\mathrm{Ha} \mathrm{Oh}^{4,5}$ \\ ${ }^{1}$ The Armed Forces Daejeon Hospital, \\ Daejeon; ${ }^{2}$ PLUS Aesthetic Surgery Clinic, \\ Daejeon, Korea; ${ }^{3}$ Seoulige Aesthetic \\ Hospital, Shanghai, China; ${ }^{4}$ Department \\ of Plastic and Reconstructive Surgery, \\ Chungnam National University Hospital, \\ Daejeon; ${ }^{5}$ Clinical Anatomy Institute, \\ Chungnam National University Hospital, \\ Daejeon, Korea
}

\begin{abstract}
This article was presented as a free paper at Aesthetic plastic surgery 2013 on April 28, 2013 in Seoul, Korea.
\end{abstract}

This research was supported by Basic Science Research Program through the National Research Foundation of Korea (NRF) funded by the Ministry of Science, ICT and Future Planning (2013R1A1A1057928).

The authors would like to thank Dong-Su Jang, MFA, (Medical Illustrator, Seoul, Korea) for his help with the illustrations.

No potential conflict of interest relevant to this article was reported.
Background Correction of a bulbous tip is a difficult procedure in Asians, because their lower lateral cartilage is relatively small and structurally weak to support the thick skin soft tissue envelope (SSTE). Therefore, lower lateral cartilage manipulation alone yields inadequate bulbous tip correction. In this study, authors aim to provide a new bulbous tip definition reflecting nasal tip SSTE and categorization with a suitable surgical procedure.

Methods One hundred sixty-three patients with tip rhinoplasty between January 2009 and December 2012 were studied who had a tip lobular width greater than $60 \%$ of the alar base width. Depending on cartilage size and characteristics of the nasal tip superficial musculoaponeurotic system (SMAS) with SSTE thickness, the following classifications were made: Group I: thin SSTE with a large lower lateral cartilage, Group II: thick SSTE with a small lower lateral cartilage, Group Ila: thick SSTE with loose SMAS, and Group Ilb: thick SSTE with dense SMAS. We evaluated the degree of surgical improvement by comparing pre- and postoperative photographs.

Results After comparing pre- and postoperative photos, we observed improvements in tip bulbosity by $11.7 \%$ in Group I $(n=41), 11.9 \%$ in Group Ila $(n=64)$, and $7.1 \%$ in Group $\mathrm{Ilb}(\mathrm{n}=58)$.

Conclusions In Asians, nasal tip bulbosity is often due to excess SSTE. Therefore, a bulbous tip should be defined and evaluated based on its underlying SSTE. Adequate soft tissue resection in addition to lower lateral cartilage support and manipulation are warranted to achieve a refined tip.

Keywords Asians, Bulbous nose, Nasal tip plasty, Rhinoplasty, Skin soft tissue envelope

\section{INTRODUCTION}

A bulbous nasal tip is commonly seen in Asians; its correction represents a significant portion of Asian rhinoplasties. Bulbous tip correction in Caucasians mainly aims at reducing excess lower lateral cartilage and performing suture techniques on well-developed lower

Received: Sep 30, 2014 Revised: Oct 13, 2014 Accepted: Oct 15, 2014

Correspondence: Jae Yong Jeong PLUS Aesthetic Surgery Clinic,

203 Daedeok-daero, Seo-gu, Daejeon 302-829, Korea.

E-mail: dogearjeong@naver.com

Copyright @ 2014 The Korean Society for Aesthetic Plastic Surgery.

This is an Open Access article distributed under the terms of the Creative Commons Attribution Non-Commercial License (http://creativecommons.org/licenses/by-nc/3.0/) which permits unrestricted non-commercial use, distribution, and reproduction in any medium, provided the original work is properly cited. $\quad$ www.e-aaps.org lateral cartilage [1-4]. Asians with a bulbous tip have a small, weak lower lateral cartilage and nasal septum $[5,6]$. Therefore, there is a limit to how much one can project the lower lateral cartilage and push against the nasal tip skin and soft tissue, the so-called SSTE (skin soft tissue envelope). In addition, there is a high incidence of nasal SMAS (superficial musculoaponeurotic system) thickening [1]. Thus, reshaping and repositioning the lower lateral cartilage are often masked by a thick nasal skin envelope $[1,2,7,8]$.

In Asians, one must incorporate SSTE thickness when defining a bulbous tip. A simple surface evaluation of a broad or large nasal tip is insufficient to describe a bulbous tip. Classification using cartilage shape was previously introduced in the literatures $[2,3,5]$, but such a definition provides a limited description of the actual appearance of the bulbous tip. Some authors have shown interest in SSTE and tried to explain "bulbous" as the unfavorable shadowing 
of the tip contour [1,9], whereas others have tried to explain "bulbous tip" using optical illusions in relation to other nasal components [2]. However, a clear definition and classification of a bulbous tip has not been established. For these reasons, the authors present a quantitative definition reflecting the SSTE and grouped bulbous nasal tip, focusing on the anatomical characteristics of SSTE, especially SMAS, with the application of appropriate operative and adjunctive techniques. Moreover, the aim of the present study is to investigate post-surgical outcomes of each subclassified
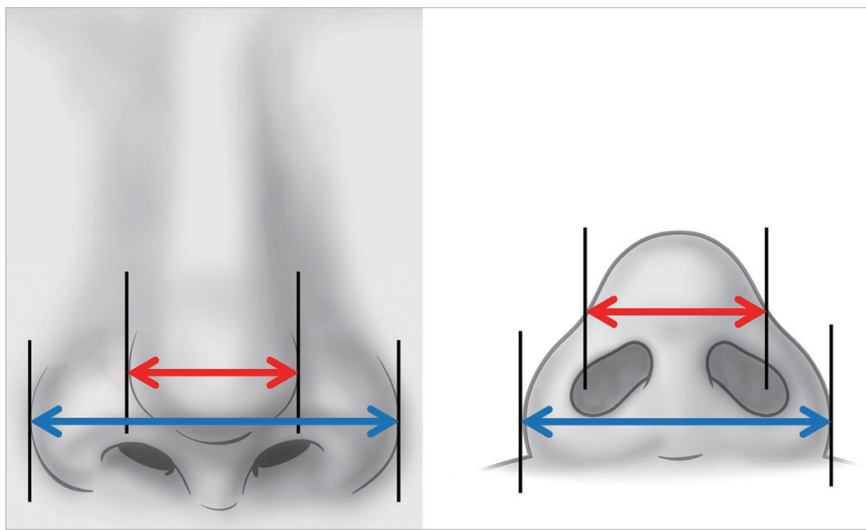

Fig. 1. Tip lobule width: alar width is calculated as the bulbosity index (B.I.). B.I. over 0.6 is defined as a bulbous tip. Red line: Tip lobule width; Blue line: Alar width. bulbous group.

\section{METHODS}

From January 2009 to December 2012, primary tip rhinoplasty patients had the tip lobule width and alar base width measured from frontal or basal view pictures. The ratio of the tip lobule width to the alar basal width was named the bulbosity index (B.I.). A bulbous tip was defined using this B.I. index as being greater than or equal to 0.6 , through peer review (Fig. 1). Patients fulfilling this criterion (163) underwent bulbous tip rhinoplasty surgery. Of these patients, 134 were female and 29 were male, with a mean age of 26 years.

We segregated the patients into three groups according to their preoperative bulbous nasal tip SSTE characteristic and the size of their lower lateral cartilage. Group I is characterized by a bulbous shape resulting from a relatively thin SSTE compared to the large lower lateral cartilage. Group II is characterized by a bulbous tip appearance resulting from thick SSTE. Group II patients are subdivided according to their SMAS characteristics. Patients with a loose SMAS are considered Group IIa, and patients with a firm SMAS are considered Group IIb (Fig. 2, Table 1). We then applied the following surgical procedures that were specifically designed to target patients in each group (Table 2).

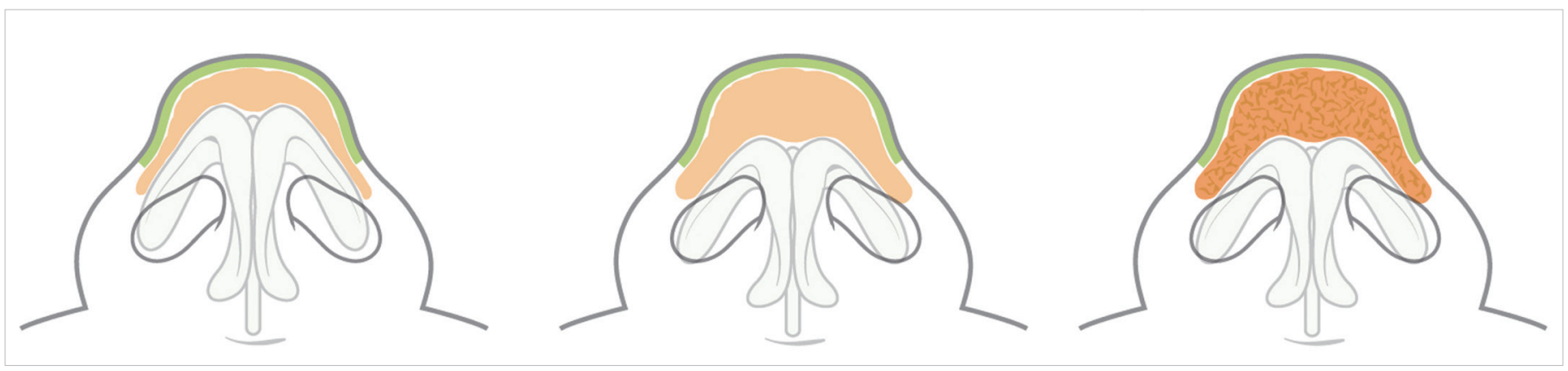

Fig. 2. A bulbous nasal tip is divided into Groups I and II; the latter is subdivided into Groups Ila and IIb.

Table 1. Characteristics of each group

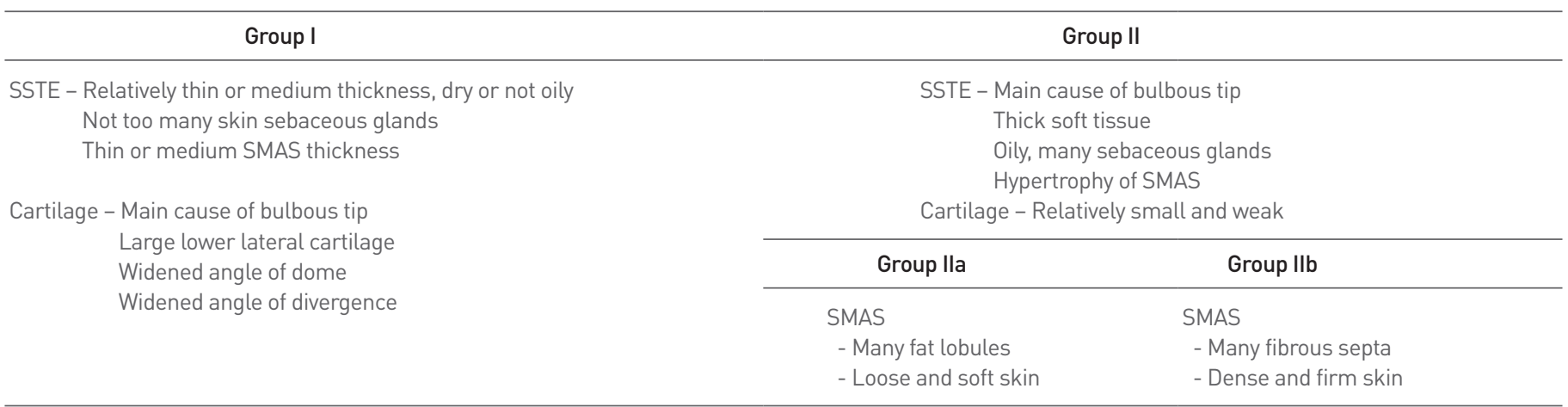


Table 2. Operative and adjunctive procedures

\begin{tabular}{|c|c|c|}
\hline Group I & \multicolumn{2}{|c|}{ Group II } \\
\hline & Group Ila & Group Ilb \\
\hline
\end{tabular}
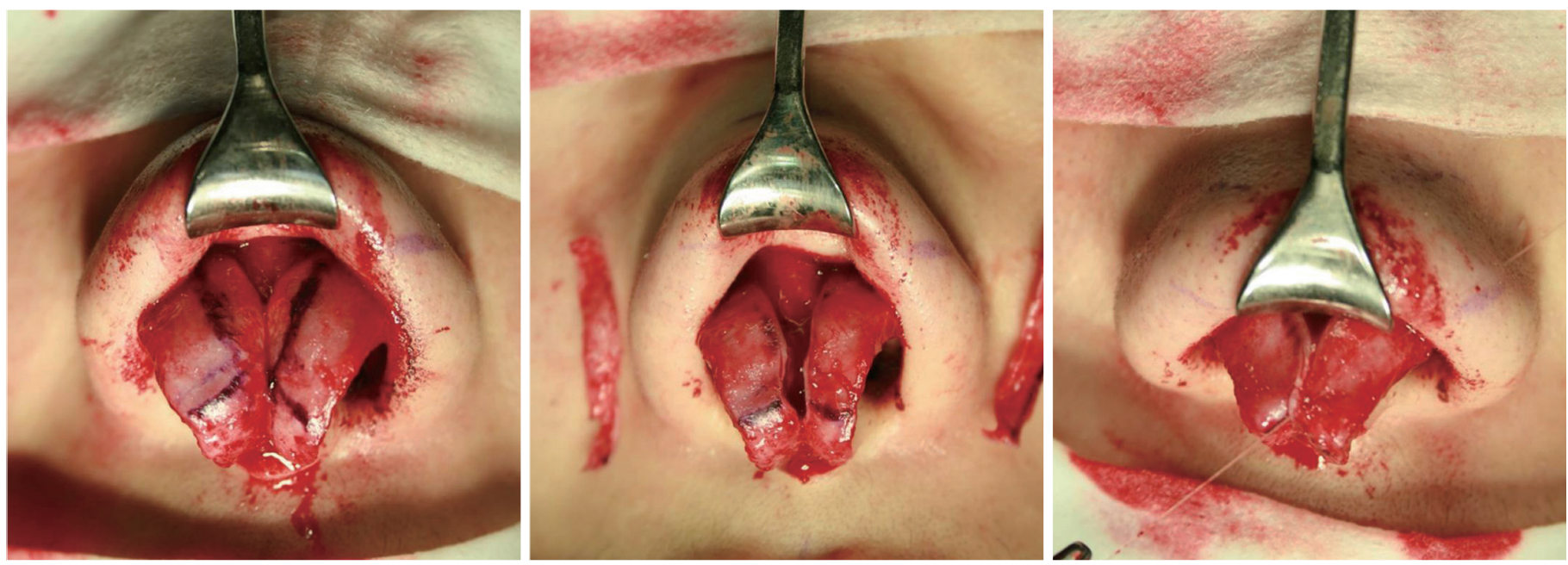

Fig. 3. Cephalic resection and suturing are applied to Group I.

\section{Surgical method}

During the preoperative physical examination, overall soft tissue volume, sebaceous content, firmness and elasticity of the SSTE, and the size of the lower lateral cartilage were evaluated, and the surgical procedure was planned. The operation was done under local anesthesia with intravenous anesthesia using midazolam.

Columella flap elevation was achieved by performing an inverted V-shaped transcolumella incision and infracartilagenous marginal incision. The tip rhinoplasty method is described below.

\section{Group I operation}

Group I patients have a relatively large lower lateral cartilage relative to their thin SSTE. Therefore, it is crucial to preserve sufficient SSTE. An incision was made from the subSMAS and supraperichondrial plane of the lower lateral cartilage, through the cartilaginous dome, and to the subperiosteal plane at the keystone area to achieve flap elevation.

The retaining ligament in the scroll area was released to partially separate the lower lateral cartilage from the upper lateral cartilage.
Cephalic resection of the lower lateral cartilages was performed while preserving 6 to $7 \mathrm{~mm}$ of lateral crus and 4 to $5 \mathrm{~mm}$ of middle crus. Transdomal, interdomal, and lateral crural spanning sutures were used to achieve an ideal dome shape. A cartilage graft from the ear, septum, or rib was extracted to constitute a supporting framework, if necessary. The soft tissue resection procedure, such as SSTE defatting, was minimized and an onlay or shield graft was added for more tip definition. Care was given to smooth the edges of the tip grafts so that no sharp edges would project through the nasal skin envelope (Fig. 3).

\section{Group II operation}

At the tip and supratip regions, intraSMAS dissection was made to separate the SMAS into the superficial SMAS (attached to the skin) and the deep SMAS (attached to the lower lateral cartilage). Afterward, partial SMASectormy of the deep SMAS was performed (Fig. 4).

It is important to maintain a constant thickness of the elevated flap so that it does not affect the subdermal plexus circulation of the tip envelope. The excised SMAS and soft tissue were preserved 

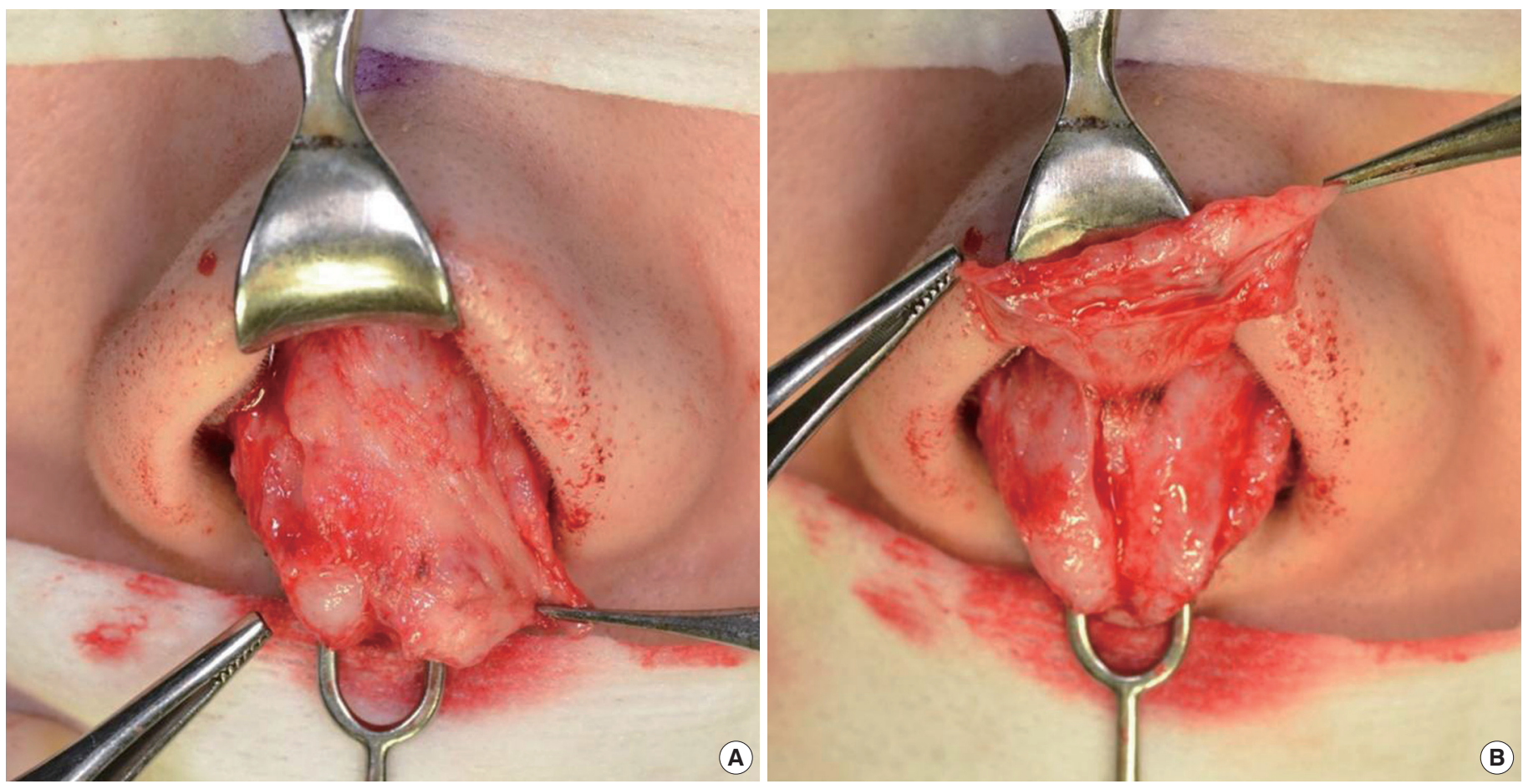

Fig. 4. (A) The nasal envelope is elevated with intraSMAS dissection. Part of the SMAS that is to be resected enbloc is the lower SMAS that is attached to the lower lateral cartilage. (B) SMASectomy is completed with supraperichondrial dissection. Resected soft tissue is stored and grafted later if needed to shape the tip.

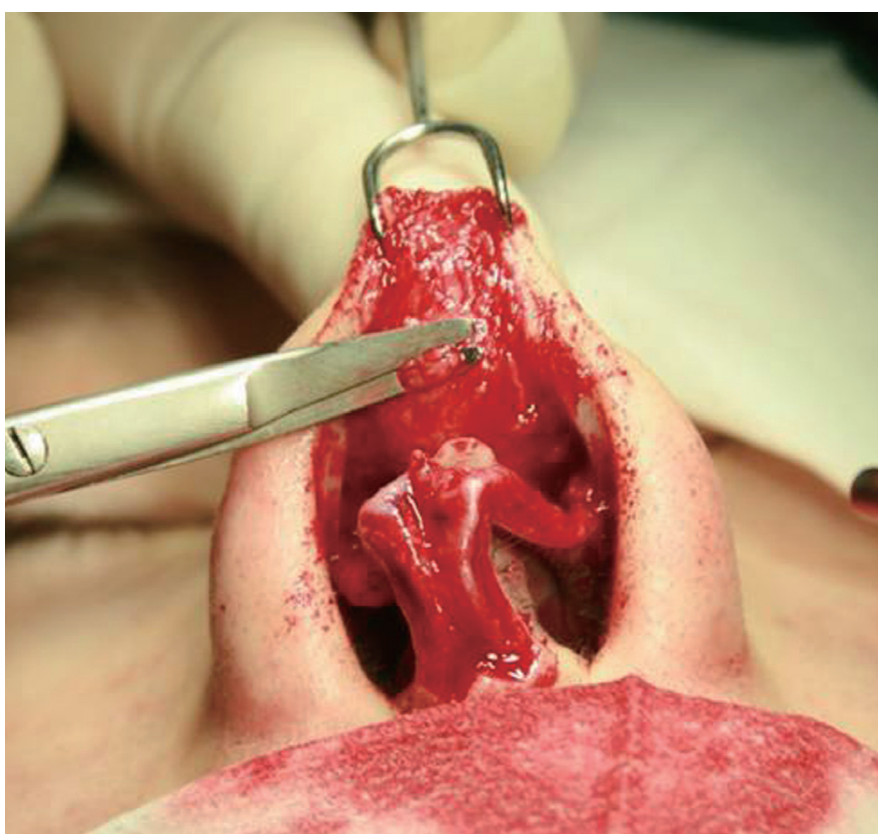

Fig. 5. Additional defatting is done for Group Ila.

for possible use as soft tissue grafts, if necessary. The tip framework was achieved using ear, septum, or rib cartilage to support the tip. Subsequently, tip suturing and cartilage grafting were performed to refine the nasal tip.

\section{Group IIa operation}

Flap elevation can be done more easily for patients with loose SMAS than for group IIb patients. Also, it is easier to redrape the nasal soft tissue envelope to cover the cartilage frame and to achieve additional defatting on the elevated SSTE. As in Group I, the tip was refined by projecting the lower lateral cartilage against the defatted soft tissue of the nasal tip (Fig. 5).

\section{Group IIb operation}

In the case of dense SMAS, the skin envelope is inelastic due to the high amount of fibrotic tissue (Fig. 6). Therefore, the silhouette of the projected lower lateral cartilage does not reflect through SSTE, and the postoperative result is often unsatisfactory. Moreover, there is a higher possibility of prolonged edema. For these reasons, it is prudent to inform the patient about the difficulty of correcting their particular bulbous tip and postoperative sequelae during the preoperative evaluation. Also, thorough postoperative care, such as gentle pressure dressing, steroid injections, and hyaluronidase injections, can help achieve optimal results.

\section{RESULTS}

Of the bulbous tip rhinoplasty patients studied by follow-up observations from 3 to 24 months, the mean follow-up period was 12.8 months. One hundred twenty-two patients were diagnosed with 
thick SSTE, and thus categorized in either Group IIa or IIb, which accounted for $74.8 \%$ of the total bulbous tip rhinoplasty patients (Table 3).

The B.I. was improved by $11.7 \%$ in Group I $(n=41)$, from 0.68 to $0.60,11.9 \%$ in Group IIa $(\mathrm{n}=64)$, from 0.67 to 0.59 , and $7.1 \%$ in Group IIb ( $\mathrm{n}=58)$, from 0.71 to 0.66 , respectively (Table 4 ).

Comparisons between pre- and postoperative. B.I. index affirms that Group IIa saw improvements similar to Group I. However, bulbous tip correction is challenging in Group IIb (Fig. 7-9).

Delayed wound healing in columella and vestibular incision sites was observed in five male patients, who were smokers, in Group

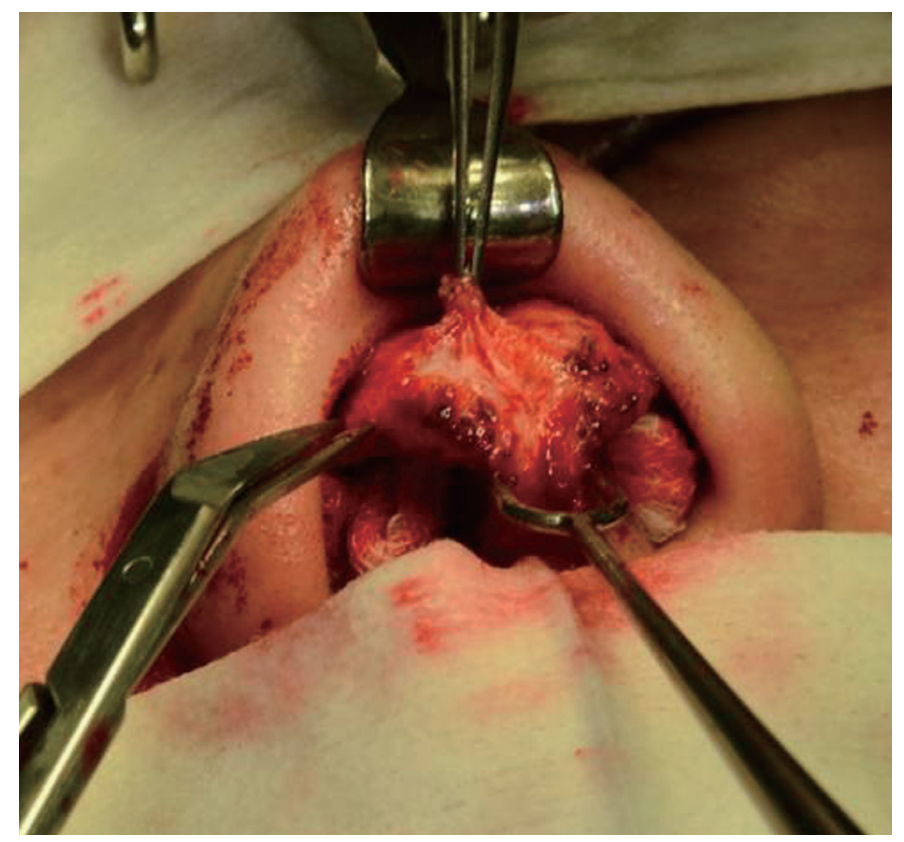

Fig. 6. A greater amount of subcutaneous fibrous fatty tissue is seen in Group Ilb. Thick SMAS with dense SSTE needs careful resection of the SMAS. This group has adverse healing of the resected dead space with frequent recurrence of a thick, enveloped bulbous tip.
IIb, but ultimately, the wound healed without further complications. There were no cases of nasal tip SSTE necrosis. Seven patients in Group IIb showed signs of undercorrection, so they underwent triamicinolone and hyaluronidase injections during their follow-up observations. Four revision surgeries were needed one patient in Group IIa and three patients in Group IIb due to a bulbous nasal tip

\section{DISCUSSION}

The shape of the nasal tip is a combination of the underlying lower lateral cartilage framework and the overlying soft tissue envelope $[1,2]$. To create an aesthetically pleasing and refined tip, a surgeon must not only reshape the underlying framework but also debulk the overlying soft tissue envelope. In addition, management of patient expectations based on their anatomy is important for achieving patient satisfaction.

Table 3. Number of patients in each group

\begin{tabular}{lcc}
\hline Group & No. of patients & Percentage (\%) \\
\hline Group I & 41 & 25 \\
Group Ila & 64 & 39 \\
Group Ilb & 58 & 36 \\
Totals & 163 & 100 \\
\hline
\end{tabular}

Table 4. Comparison of pre- and postoperative bulbosity by B.I. and postoperative B.I. improvement (\%)

\begin{tabular}{lccc}
\hline & Group I & Group Ila & Group Ilb \\
\hline Preop. B.I. ${ }^{\text {al }}$ & 0.68 & 0.67 & 0.71 \\
Postop. B.l. & 0.60 & 0.59 & 0.66 \\
B.I. $^{\text {b) }}$ improvement & 11.70 & 11.90 & 7.10 \\
\hline
\end{tabular}

${ }^{a}$ B.I., bulbosity index, is calculated as the ratio of the tip lobule width to alar base width; ${ }^{b}$ B.I. improvement between pre- and postoperative B.I. (\%).
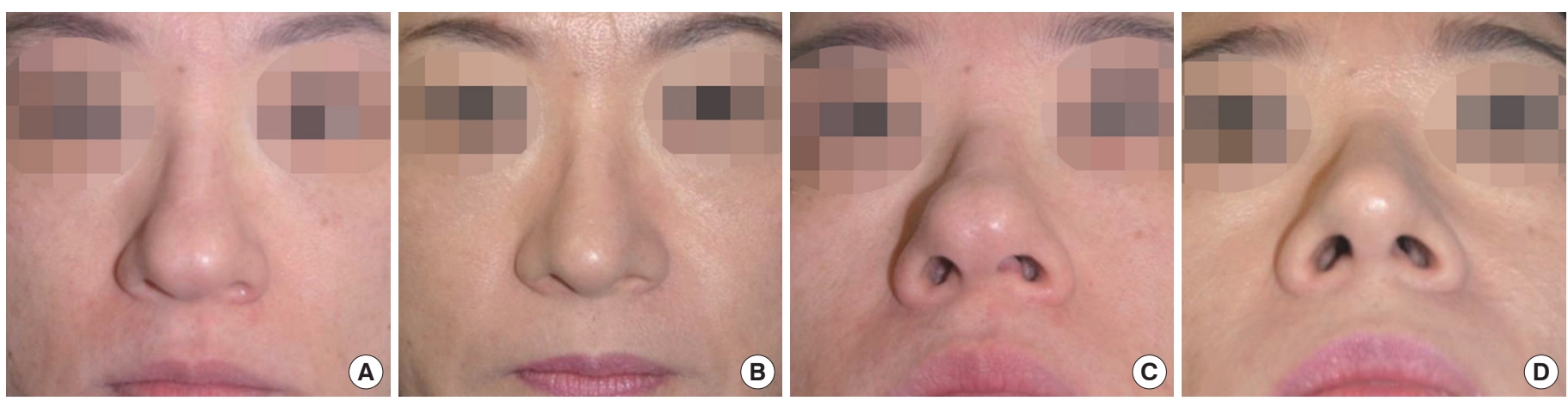

Fig. 7. Group I patient: Thin SSTE and thin SMAS, 42-year-old female, lower lateral cartilage reshaping via medial and lateral crus resection, transand interdomal sutures, and lateral crural spanning sutures. Improvements of approximately $11.4 \%$, achieving B.I. of 0.62 following surgery compared to 0.70 before surgery. (A) Preoperative frontal view, (B) Postoperative frontal view, (C) Preoperative basal view, and (D) Postoperative basal view. 

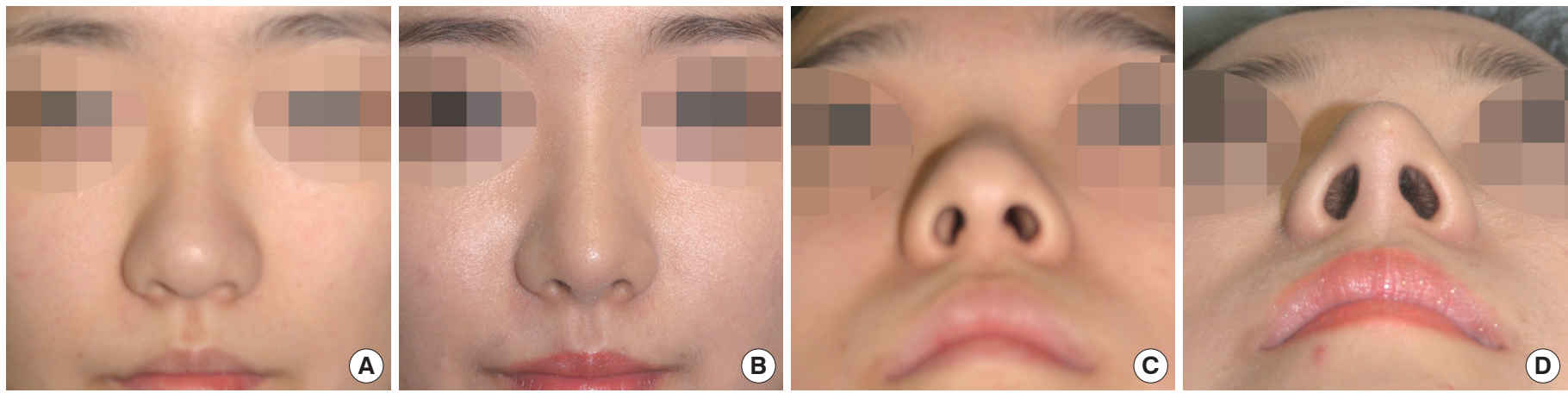

Fig. 8. Group Ila patient: Thick SSTE with loose SMAS, 24-year-old female, cartilage manipulation via enbloc SMASectomy and additional defatting. There was an improvement of approximately $15.5 \%$, achieving a B.I. of 0.60 following surgery compared to 0.71 before surgery. (A) Preoperative frontal view, (B) Postoperative frontal view, (C) Preoperative basal view, and (D) Postoperative basal view.
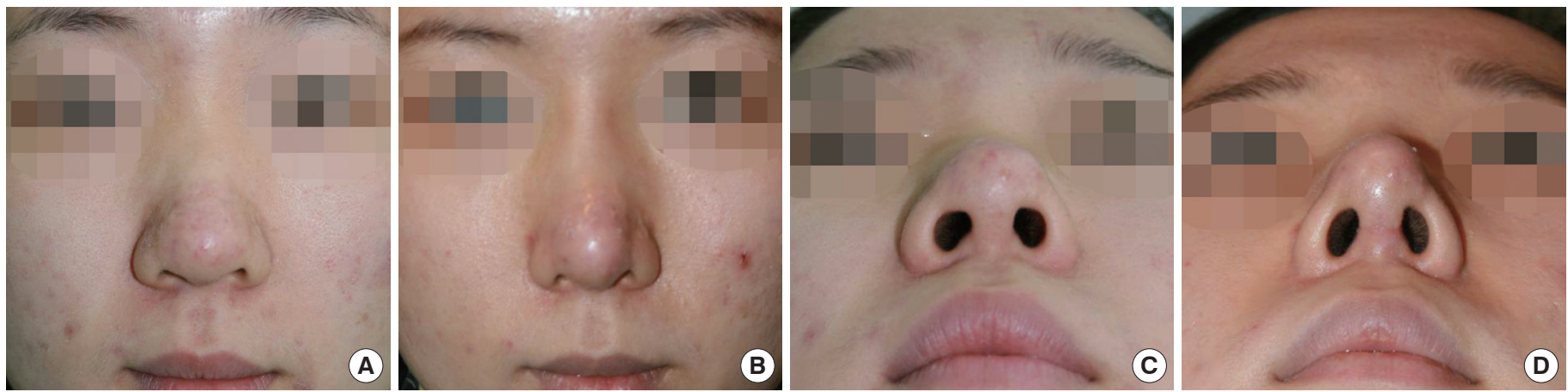

Fig. 9. Group IIb patient: Thick SSTE and dense SMAS, 22-year-old female, cartilage manipulation via enbloc SMASectomy. Triamcinolone was injected at 3 and 6 months of follow-up due to a recurrent bulbous tip. There was an improvement of approximately $7.4 \%$, achieving a B.I. of 0.63 following surgery compared to 0.68 before surgery. (A) Preoperative frontal view, (B) Postoperative frontal view, (C) Preoperative basal view, and (D) Postoperative basal view.

Generally, the patient is thought to have a bulbous tip when he/ she has an undefined and broad tip shape. Until now, the definition of a bulbous tip has largely depended on the shape and size of the cartilage from a basal view $[2,3,5]$. However, this method of evaluation is limited to the components of the nasal tip but their relationships are not considered. This is especially true in Asians where the cause of a bulbous tip is in the SSTE, not in the cartilage. In such cases, the cartilage is not expressed due to a thick envelope. Some have stated that the tip contour appears bulbous because of the relative size difference in the nasal dorsum, alar, and alar base [2,9], but this is an inadequate definition, lacking quantitative analysis. Also, the conventional definition does not accurately portray the shape of the tip lobule that one would see.

Therefore, the aim of the present study is to define tip bulbosity, reflecting the actual appearance of the tip contour, including the SSTE, regardless of the applied surgical methods. As a result, a bulbous tip is defined as an alar basal width to tip lobule width ratio greater than or equal to 0.6. One of the advantages of this method is that the scale of the photo is not necessary, because only the relative size differences are compared.
Most bulbous tip correction methods that have been introduced, such as cartilage resection, reduction, grafts, and suture techniques, are used for Caucasians with a well-developed lower lateral cartilage [2-4]. However, Asians with a bulbous tip have an under-developed lower lateral cartilage, and there is a high incidence of nasal SMAS thickening $[1,6]$, so reshaping and repositioning the lower lateral cartilage alone cannot generate satisfactory results in Asians.

The thickness of the soft tissue envelope acts as a limiting factor in solving the bulbous nasal tip problem. However, regardless of the level of difficulty that the SSTE presents in correcting a bulbous tip, the hypertrophic thickening of the SMAS, which is the underlying cause of a thick SSTE around the nasal tip, is the most important factor in the correction of a bulbous tip $[1,7,8]$.

Some surgeons have negative opinions about tip SMASectomy, recognizing it as a potential threat to tip circulation [2]; there are scarce reports that discuss SSTE management [4]. However, investigating the literatures on this topic, the blood supply to the tip is known to flow from the lateral nasal and dorsal nasal arteries to the inner SMAS, creating an abundant subdermal plexus around the tip $[1,7,8,10,11]$ (Fig. 10). Thus, as long as the subdermal plexus 


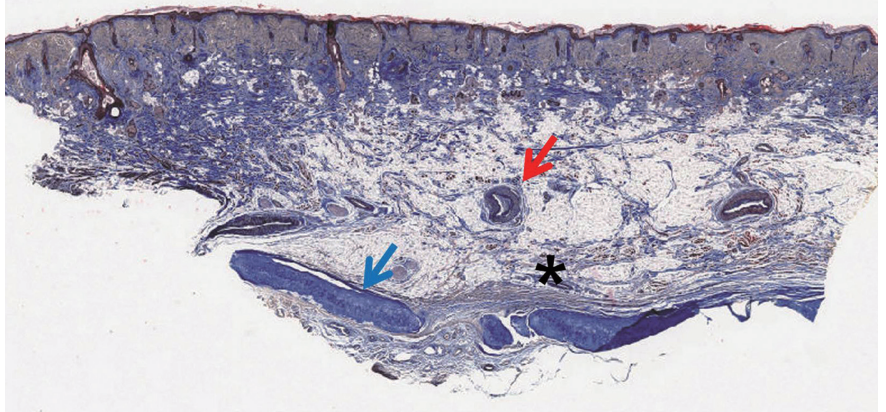

Fig. 10. Cross-section of the nose, showing major vessels passing into the SMAS layer. Red arrow: Lateral nasal artery, Asterisk: Nasal SMAS. Blue arrow: Lower lateral cartilage (hematoxylin and eosin stain).

is not damaged, partial enbloc SMASectomy can safely be done.

Based on clinical experience and cadaver studies, a thickened SMAS is composed of loose SMAS with a high number of fat lobules and firm fibrotic septa, forming a dense SMAS (Fig. 11). Different skin types in terms of skin thickness, elasticity, texture, and sebaceous gland number vary among patients; an underlying cause of a thick SMAS $[1,8,12]$. SSTE is a key factor of SMAS characteristics [1]. Just as different people have different skin types, differences in SMAS types must be taken into account and approached accordingly.

Therefore preoperative evaluation through palpation serve important roles. As a precaution to disfigurement or secondary deformity from the envelope, it is crucial to provide sufficient preoperative surgical planning and counseling to discuss the limitations of the surgery, which later helps inform the need for additional operations and a realistic prediction of surgical results.

For these reasons, the patients were grouped according to their SSTE and cartilage characteristics. Group I patients whose tip bulbosity came from their large lower lateral cartilage, underwent rather radical methods such as transection and resection, and tip shaping is done through more conservative methods, such as suturing and grafting, which serve to reduce the angle of divergence and domal definition. Cartilage manipulation is the cornerstone of tip rhinoplasty, so a framework is needed to support the various tips in different groups.

In Group II, the main cause of tip bulbosity is SSTE, and hypertrophic thickening of the nasal SMAS layer is considered to be the underlying cause of the thick SSTE. The SMAS is a scaffold comprised of collagen fibers, elastic fibers, and fat cells, and has a fibrous network connecting dermis and facial muscle $[1,8,12,13]$. For Group IIa, more fat lobules are observed in this area, which leads to loose and less-dense fibrous septa, and thus less hardened skin. Therefore, additional defatting is not needed, and the envelope is easily redraped to fit the frame. Postoperative edema is significant in the
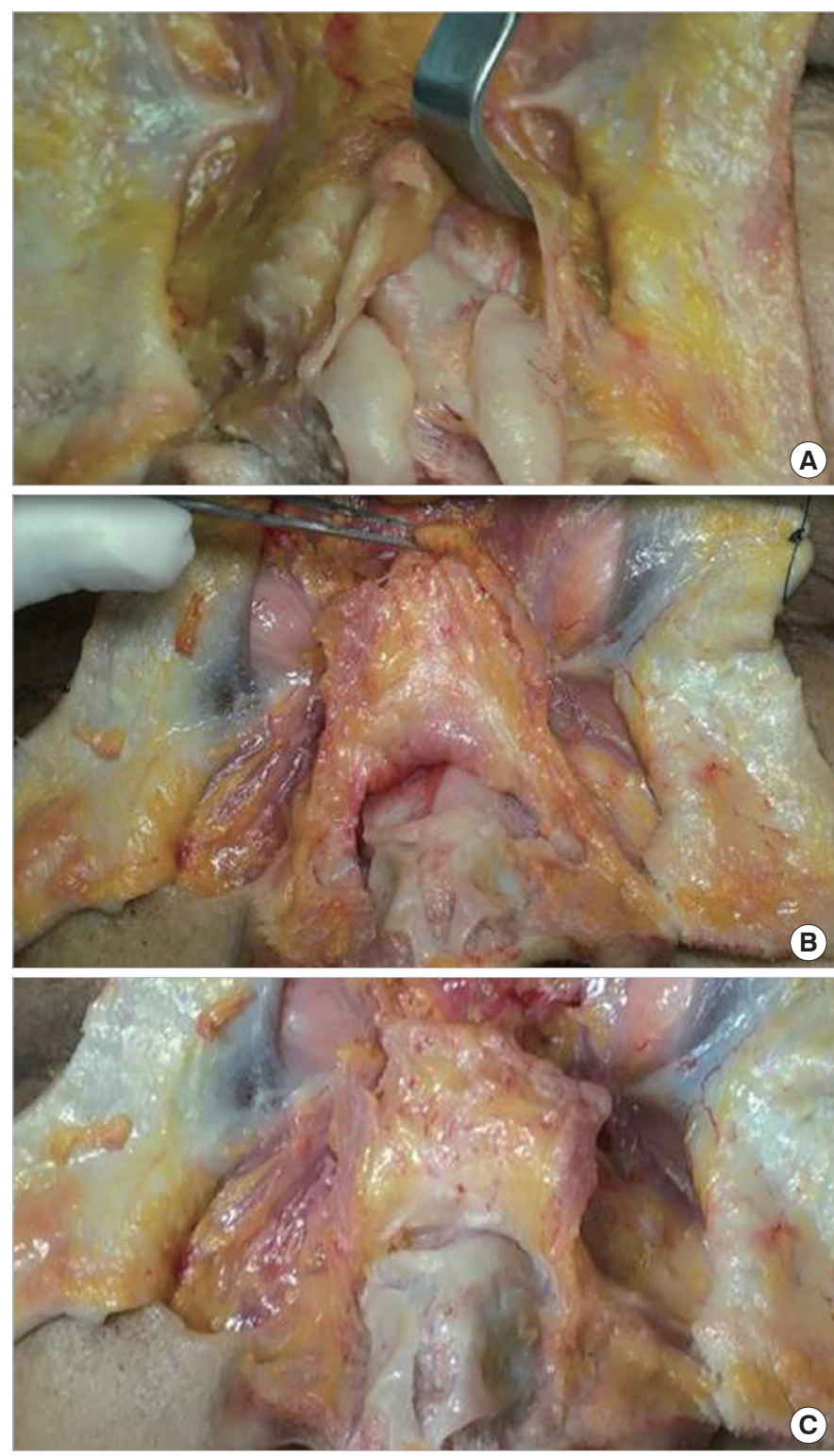

Fig. 11. Comparison of nasal SMAS in a cadaveric study. (A) Thin SMAS, (B) Thick SMAS with loose fat lobules, and (C) Thick SMAS with firm fibrotic septa.

case of loose SMAS, regardless of its thickness. Thus, postoperative results are favorable. However, Group IIb patients, with a dense SMAS, are particularly challenging cases for bulbous tip correction, because, unlike patients with loose SMAS, the envelope of the dense SMAS is firm, and redraping is not easily done, so external expression of the tip shape is difficult to achieve by SMASectomy. If this problem is solved by aggressive thinning of the SSTE, there is a danger of subdermal plexus injury. Follow-up observations have shown that it is difficult to predict results due to the adverse healing characteristics of dense SMAS and prolonged edema, which increase fibrosis and hypertrophic scar formation of the soft tissue $[1,7,10]$. For these reasons, it is hard to generate consistent results 
in Group IIb. It is important to perform sufficient preoperative consultations with patients and provide sufficient follow-up periods. If the bulbosity recurs adjunctive procedures such as triamcinolone or hyaluronidase injections can be considered.

In the present study, 12 patients experienced a recurrent bulbous tip even with the sufficient removal of SMAS; four of these patients underwent revision surgery.

A bulbous nasal tip is a common problem that surgeons face in the field of Asian rhinoplasty. However, lacking a definition for a bulbous tip with a thick SSTE, which is often observed in Asian populations, it is hard to correct a bulbous nasal tip. A new definition of a bulbous tip using the tip lobule width to the alar basal width ratio is suggested, which enables the quantitative analysis of preoperative bulbosity and improvements after surgery. Moreover, the categorization of a bulbous tip allows for a more effective correction and predictable results. Since the nasal cartilage frame is transformed into the outer contour by SSTE, it shapes the tip and provides surface definition. So it is hard to sustain a fine tip without the proper handling of nasal SMAS, which determines the SSTE characteristics. Especially for Asian bulbous tip patients, who tend to have a thick SSTE, the surgeon's ability to predict limitations in the surgical correction regarding the envelope is crucial to obtaining satisfactory cosmetic results.

\section{REFERENCES}

1. Azizzadeh B, Murphy MR, Johnson CM, et al. Master techniques in rhinoplasty. Philadelphia: Elsevier Saunders; 2011.
2. McKinney P. Management of the bulbous nose. Plast Reconstr Surg 2000;106:906-17.

3. Rohrich RJ, Adams WP Jr. The boxy nasal tip: classification and management based on alar cartilage suturing techniques. Plast Reconstr Surg 2001;107:1849-63.

4. Mao GY, Yang SL, Zheng JH, et al. Aesthetic rhinoplasty of the Asian nasal tip: a brief review. Aesthetic Plast Surg 2008;32:632-7.

5. Kang JS. Plastic surgery. Seoul: Koonja; 2004.

6. Korean Society of Rhinoplastic Surgeons. Current trends in Asian rhinoplasty: operative guide. Seoul: Koonja; 2011.

7. Steele NP, Thomas JR. In: Stucker FJ, Souza C de, Kenyon GS, et al., editors. Rhinoplasty and facial plastic surgery. New York: Springer; 2009. p. 5-12.

8. Jewett BS, Baker SR. In: Baker SR, editor. Principles of nasal reconstruction. New York: Springer; 2011. p. 13-22.

9. Toriumi DM. New concepts in nasal tip contouring. Arch Facial Plast Surg 2006;8:156-85.

10. Toriumi DM, Mueller RA, Grosch T, et al. Vascular anatomy of the nose and the external rhinoplasty approach. Arch Otolaryngol Head Neck Surg 1996;122:24-34.

11. Jeong JY. Tripod framework rebuilding in Asian nose: tip plasty using alar advancement technique, J Korean Soc Aesthetic Plast Surg 2010; 16:125-38.

12. Letrourneau A, Daniel RK. The superficial musculoaponeurotic system of the nose. Plast Reconstr Surg 1988;82:48-57.

13. Ghassemi A, Prescher A, Riediger D, et al. Anatomy of the SMAS revisited. Aesthetic Plast Surg 2003;27:258-64. 